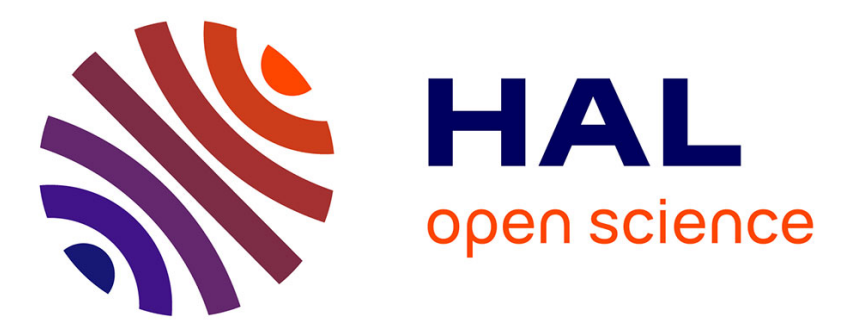

\title{
Tumor immunosurveillance and immunotherapies: a fresh look from intravital imaging
}

Morgane Boulch, Capucine L. Grandjean, Marine Cazaux, Philippe Bousso

\section{To cite this version:}

Morgane Boulch, Capucine L. Grandjean, Marine Cazaux, Philippe Bousso. Tumor immunosurveillance and immunotherapies: a fresh look from intravital imaging. Trends in Immunology, 2019, 40 (11), pp.1022-1034. 10.1016/j.it.2019.09.002 . pasteur-02944694

\section{HAL Id: pasteur-02944694 \\ https://hal-pasteur.archives-ouvertes.fr/pasteur-02944694}

Submitted on 21 Sep 2020

HAL is a multi-disciplinary open access archive for the deposit and dissemination of scientific research documents, whether they are published or not. The documents may come from teaching and research institutions in France or abroad, or from public or private research centers.
L'archive ouverte pluridisciplinaire $\mathbf{H A L}$, est destinée au dépôt et à la diffusion de documents scientifiques de niveau recherche, publiés ou non, émanant des établissements d'enseignement et de recherche français ou étrangers, des laboratoires publics ou privés.

\section{(1) (1) $\$$}

Distributed under a Creative Commons Attribution - NonCommercial - NoDerivatives $\mid 4.0$ 


\title{
Tumor immunosurveillance and immunotherapies: a fresh look from intravital imaging
}

\author{
Morgane Boulch $^{1,2}$, Capucine L. Grandjean ${ }^{1}$, Marine Cazaux ${ }^{1,3}$ \\ and Philippe Bousso ${ }^{1}$
}

Keywords: Tumor immunity, immunosurveillance, immunotherapy, intravital imaging, twophoton microscopy

${ }^{1}$ Dynamics of Immune Responses Unit, Equipe Labellisée Ligue Contre le Cancer, Institut Pasteur, INSERM U1223, 75015 Paris, France.

${ }^{2}$ Master de Biologie, École Normale Supérieure de Lyon, Université Claude Bernard Lyon I, Université de Lyon, 69342 Lyon Cedex 07, France.

${ }^{3}$ University Paris Diderot, Sorbonne Paris Cité, Cellule Pasteur, rue du Dr Roux, 75015 Paris, France.

Correspondence to: philippe.bousso@pasteur.fr 
Understanding the complex interactions between the immune system and the tumor microenvironment is an essential step toward the rational development and optimization of immunotherapies. Several experimental approaches are available to tackle this complexity but most are not designed to address the dynamic features of immune reactions including cell migration, cellular interactions and transient signaling events. By providing a unique means to access these precious parameters, intravital imaging offers a fresh look at intratumoral immune responses at the single-cell level. Here, we discuss how in vivo imaging sheds light on fundamental aspects of tumor immunity and helps elucidate the mode of action of immunotherapies. We conclude by discussing future developments that may consolidate the unique contribution of intravital imaging for our understanding of tumor immunity.

\section{Decoding mechanisms of tumor immunity}

Recent years have witnessed multiple successes for tumor immunotherapies. Despite these breakthroughs, we are far from fully understanding how the immune system acts within the complexity of the tumor microenvironment (see Glossary) and how to best harness immune cells to eradicate distinct types of tumors. To date, most of the available immunotherapies are only effective in a fraction of patients and in a subset of cancers. A full mechanistic understanding is therefore essential for increasing the efficacy of these treatments, for developing new strategies or for combining them together. Multiple approaches are available to conduct these mechanistic studies. In preclinical models, manipulation of immune subsets (using knock-out models or antibody-based depletion for example) or genetic manipulation of tumor cells are routinely used. From human samples, high dimensional flow cytometry or single-cell RNA sequencing are powerful technologies to establish important correlations and to suggest putative mechanisms that could be further explored. These types of approaches have 
provided important insights into the differential modes of action of blocking $\mathrm{T}$ cell co-receptors relevant to immunotherapies, such as via anti-CTLA-4 (cytotoxic T lymphocyte-associated protein 4) or anti-PD-1 (programmed cell death 1) [1]. Most of these strategies however have not directly addressed the most dynamic aspects of immune reactions such as intratumoral cell migration, establishment of cell-cell interactions and any associated signaling events. Understanding how these dynamic events are modulated by immunotherapies is likely to help unlock some of the secrets behind their modes of action in vivo.

Intravital imaging with single-cell resolution is a technique of choice to address many of these questions in vivo and, in the recent years, has helped identify a number of previously unappreciated mechanisms operating in the tumor microenvironment. Here, we provide an overview of models and tools currently available for in vivo imaging. We next review how in vivo imaging sheds light on fundamental aspects of tumor immunosurveillance and immunosuppression. We also address how dynamic imaging helps elucidate the mode of action of immunotherapies and discuss future technical developments that will reinforce the use of intravital imaging in preclinical models of cancer.

\section{Using intravital imaging to assess tumor immunity}

At the beginning of the 20th century, pioneering experiments by Elie Metchnikoff using optical microscopy demonstrated that immune reactions occurred in transparent organisms, such as Daphnia magna and starfish larvae [2]. As described elsewhere, progress in imaging technology and biological tools have reinvigorated the use of in vivo imaging in the last few decades $[3,4]$. To date, spinning-disk confocal microscopy, laser scanning confocal microscopy, and two-photon microscopy are the three main high-resolution methods for intravital imaging. Each has its own benefits and drawbacks and should be chosen depending on the biological constraints. Briefly, spinning-disk confocal imaging is preferred for its rapid 
image acquisition while conventional laser scanning confocal microscopy can generate images of higher resolution. Finally, two-photon microscopy is most often used for deep tissue imaging as it relies on fluorophore excitation by low-energy infrared light.

Intravital imaging in mouse models has provided significant insights into basic immune mechanisms of lymphocyte activation or effector immune responses in a variety of contexts. Applied to preclinical models of cancer with relevant fluorescent labeling, in vivo imaging approaches can therefore offer access to numerous dynamic processes in the tumor microenvironment, with the potential to reveal new mechanisms of immune cell activity during tumor development, or during the course of tumor immunotherapies. For instance, by offering crucial information on cellular interactions, cell motility and cell signaling, intravital imaging has proven useful for identifying cellular partners as well as determining whether cells are engaged in antigen-specific interactions (Figure 1, Key Figure). The typical workflow for assessing host-tumor interactions in animal models using intravital imaging is described in Box 1. Of note, a number of in vivo imaging studies have examined the role of the tumor vasculature in influencing indirectly immune responses; we refer the reader to excellent published reviews on that topic $[5,6]$.

\section{Models and tools for intravital imaging of tumor immunity}

A large and constantly expanding toolbox is available for intravital imaging of antitumor immune responses. Both human and mouse models have been developed to microscopically explore antitumor immunity. Tumors derived from patients can be transplanted, orthotopically or ectopically, into immunodeficient mice (such as NSG (NOD scid gamma) mice) directly (patient-derived xenograft (PDX) models) or following in vitro culture (cell line-derived xenograft (CDX) models). In addition, transgenic mice engineered to develop spontaneous cancers (such as models of breast cancer in MMTV-PyMT mice [7] and B-cell lymphoma in 
E $\boldsymbol{\mu}$-myc mice [8,9]) are suitable to visualize immune responses during the course of tumor development. Strategies to fluorescently label spontaneously developing tumors have been incorporated in some models to facilitate imaging approaches. Alternatively, these spontaneous tumors can be isolated, fluorescently labeled and reinjected into secondary recipients. Finally, syngeneic mouse tumor cell lines expressing genetically-encoded fluorescent proteins or reporters have been used extensively for imaging purposes.

In tumor-bearing mice, intravital imaging has been performed in numerous anatomical sites, including the skin, lungs, mammary fatpads, digestive tract, liver, kidney, lymph nodes, bone marrow and brain. Moreover, window chambers offer the opportunity to image one particular site at multiple time points, thus giving insights into the progression of any given immune reaction in the same organism [10-15]. Alternatively, techniques using human tumor slices (e.g. from lung, ovarian tumor biopsies) obtained from patients can provide valuable information regarding immune cell behavior in the complex tumor microenvironment. Table 1 summarizes various tumor models, anatomical sites and immune cells that have been or are currently the focus of intravital imaging studies.

While a wide range of fluorescent proteins is available to label immune and tumors cells, their combination with reporters that measure functional parameters provides an additional layer of information. Examples of genetically-encoded functional reporters used to dissect host-tumor interactions are shown in Figure 2. For example, tumor cells can express probes to visualize apoptosis [16,17] and proliferation [18] in the tumor microenvironment. Tumor multicolor barcoding provides a means to study tumor clonal heterogeneity, and can give insights into tumor spatial organization $[19,20]$. In addition, immune cell activity can be tracked using gene reporters for signal transduction, including calcium signaling [17], and nuclear factor of activated T cells (NFAT) nuclear translocation [21], or for cytokine production, using for instance, interferon gamma (IFN- $\gamma$ ) or interleukin 12 (IL-12) reporters [11]. Of note, additional 
fluorescent reporters, such as oxygen probes and redox ratios, have been used in mice to image cancer development or responses to chemotherapy and could be potentially applied for the study of tumor immunity [5,22]. The continuous development of new genetically-encoded fluorescent probes to track conditions such as metabolic activity, cell phenotype and functional states can help foster new concepts in tumor immunity.

\section{Mechanisms of tumor immunosurveillance and immunosuppression}

\section{Tracking tumor infiltration.}

Tumor immunosurveillance requires migration and infiltration of effector cells into the tumor, and intravital imaging studies have helped unveil mechanisms where tumor encounters are either promoted or limited. For example, cytotoxic T lymphocytes (CTL) have been visualized: migrating, stably interacting with, and directly killing antigen-expressing subcutaneous thymoma cells in mice in vivo $[16,23,24]$. These studies have highlighted the role of cognate antigen expression on tumor cells in promoting CTL migration and intratumoral infiltration. Additionally, interactions between extracellular matrix proteins and CD44 expressed on CTL have been reported to be key determinants of interstitial migration in subcutaneous tumors [25]. A patrolling $\mathrm{T}$ cell behavior can be key to maintain cancer-immune equilibrium as shown in a melanoma model for resident memory $\mathrm{T}$ cells in the epidermis [26]. Of note, distinct effector immune cells may exhibit different immunosurveillance strategies; for example, NK cells tend to form less stable conjugates with tumor cells than $\mathrm{T}$ cells, a feature that may favor their intratumoral dissemination [27].

However, several mechanisms have also been shown to limit intratumoral CTL infiltration. Specifically, imaging studies of human lung and ovarian cancer slices revealed how stromal collagen fibers could limit $\mathrm{T}$ cell access to tumor cells [28-30]. CTL retention can also be promoted by macrophages [31] and dendritic cells (DCs) [32] in the form of unproductive stable 
interactions. Finally, CTL sequestration by apoptotic tumor cells may also limit intratumoral dissemination and reduce killing rate [16]. These studies highlight the need to delineate new $\underline{\text { strategies for promoting effective T cell-tumor cell encounters. }}$

\section{Identifying cellular partners.}

Visualizing dynamic cellular interactions can reveal putative cooperations and crosstalk between distinct immune cell types during antitumor immunity. For example, melanomaderived antigens can be captured by subcapsular macrophages in the tumor-draining lymph node and then deposited on follicular dendritic cells, thus promoting the humoral immune response [33]. At the tumor margin, in a fluorescent mouse model of spontaneous breast cancer, rare activating $\mathrm{CD}_{103}{ }^{+} \mathrm{DCs}$ can compete for $\mathrm{T}$ cell interactions with tumor-associated macrophages and favor tumor elimination [34,35]. Moreover, previously unsuspected stable NK-DC conjugates in the mouse melanoma microenvironment appeared decisive for effective antitumor responses [36].

\section{Delineating the role of immunosuppressive cells.}

Intravital imaging can also shed light on immunosuppressive mechanisms operating in the tumor microenvironment. For instance, regulatory $\mathbf{T}$ cells (Tregs) have been shown to limit $\underline{\text { the killing ability of CTLs by reducing granule exocytosis rather than by altering their capacity }}$

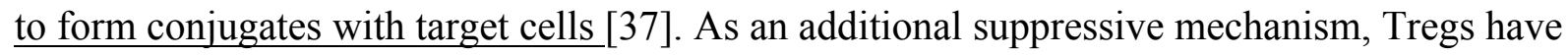
been suggested to kill DCs in tumor-draining lymph nodes, thus limiting the onset of CTL responses [38]. At the tumor site, Tregs can establish interactions with antigen-presenting cells (APCs), decreasing the surface expression of costimulatory molecules such as CD80 and CD86 on APCs and subsequently promoting CTL dysfunction [39]. Similarly, in an orthotopic pancreatic ductal adenocarcinoma model, Treg depletion promoted the accumulation of 
intratumoral DCs and their expression of costimulatory molecules, which subsequently favored prolonged contacts between CTLs and DCs [40]. Moreover, Tregs appeared to form a ring around B16 melanoma tumors that could be disrupted following cyclophosphamide administration (a chemotherapeutic agent used as a lymphodepleting, conditioning regimen for adoptive cell transfer protocols), thus favoring the infiltration of adoptively transferred CTLs [41]. Finally, the process of immunosuppressive cell recruitment can also be studied with in vivo imaging approaches. In this context, the transmigration of immunosuppressive Ly6C ${ }^{\text {lo }}$ monocytes in tumors has been reported to rely on the CX3CL1/CX3CR1 axis [14]. Altogether, these imaging studies suggest potential avenues for blocking the recruitment and activities of immunosuppressive cells in the tumor microenvironment.

\section{Uncovering spatial heterogeneity in the tumor microenvironment.}

An important outstanding question is whether immunosurveillance can occur uniformly across different tumor areas, or whether it varies due to local constraints, therefore possibly creating niches for tumor escape. Tumor-antigen specific $\mathrm{CD}^{+} \mathrm{T}$ cells have been shown to traffic differently in zones containing distinct lymphoma subclones in the bone marrow, as revealed by multicolor tumor barcoding and intravital imaging [20]. As an additional factor promoting spatial heterogeneity in the immune infiltrate, intratumoral hypoxia can be inversely correlated with $\mathrm{T}$ cell motility, especially within the core of solid tumor compared to the tumor margin [42]. Similarly, tumor-associated macrophages tracked with an Arginase-1 reporter can also exhibit distinct behaviors and morphology depending on whether they are located at the center or at the periphery of solid tumors [43]. These studies all highlight the importance of tumor $\underline{\text { microanatomy in the regulation of immune cell activity. }}$

Visualizing tumor metastasis. 
Intravital imaging can also provide mechanistic insight into tumor cell intravasation and metastasis. These processes have been visualized for example using the MMTV-PyMT mouse model of breast cancer, revealing a role for macrophages in inducing loss of vascular junctions [44]. Another study imaged how pioneer melanoma cells dynamically generate tumor microparticles in the lung vasculature when injected intravenously in mice [13]. Specifically, ingestion of these tumor microparticles by monocytes and $\mathrm{CD} 103^{+} \mathrm{DCs}$ recruited in the lungs elicited pro- and anti-metastatic effects, respectively. Finally, neutrophils could contribute to tumor metastasis through the generation of neutrophil extracellular traps (NETs) that were observed surrounding metastatic cells in the lungs [45].

As illustrated in this section, the use of intravital imaging has opened up new opportunities to reveal dynamic tumor-immune cell interactions at various stages of tumor development, to pinpoint novel cellular partnerships and to identify new putative targets that might be assessed to ideally boost immunosurveillance or circumvent immunosuppression.

\section{Toward a single-cell understanding of the mode of action of tumor immunotherapies}

With the intense efforts to develop new and efficient antitumor immunotherapies, more than ever it is essential to fully understand how these therapies precisely work in vivo. Thus, the use of intravital imaging can be decisive in confirming, clarifying or challenging other data, or perhaps preconceived ideas -- obtained most often with in vitro experiments-- about the modes of action of specific immunotherapeutic drugs.

\section{Tumor-targeting antibodies.}

Directly targeting tumors via monoclonal antibodies (mAb) can be an effective strategy to reduce tumor burden, as illustrated by the clinical use of anti-CD20, anti-EGFR (epidermal 
growth factor receptor) or anti-HER2 (human epidermal growth factor receptor 2) $\mathrm{mAb}$ for treating lymphoma, colorectal and breast cancer respectively [46]. Multiple modes of action have been proposed for $\mathrm{mAb}$, including Fc-independent effects as well as Fc-dependent

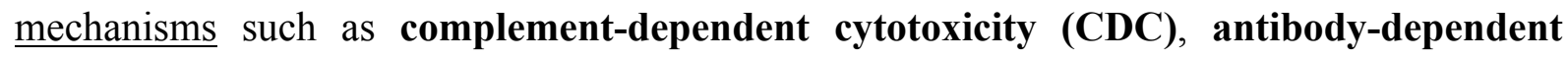
cellular cytotoxicity (ADCC) or phagocytosis (ADP) [47]. Yet, the respective contribution of each of these mechanisms in vivo is not well characterized. It is also unclear whether different mechanisms operate at distinct anatomical sites. Intravital imaging has proven to be an invaluable tool to directly address these key questions. For example, two independent studies provided evidence that ADP by Kupffer cells (KC) in the liver was a major mechanism for mAb-mediated tumor cell depletion $[48,49]$. Specifically, $\mathrm{KC}$ were visualized and shown to engulf circulating normal and malignant $\mathrm{B}$ cells within minutes following anti-CD20 administration. Similarly, KC eliminated circulating B16 melanoma tumor cells and C26 colon carcinoma cells upon mAb therapy thus preventing liver metastasis [49]. Notably, glycoengineering of $\mathrm{mAb}$ lowered the threshold for $\mathrm{KC}$-mediated depletion during anti-CD20 therapy [50]. While these studies have focused on circulating tumors, the modes of action of tumor-targeting $\mathrm{Ab}$ in solid tumors or in tumors established within lymphoid tissues remain to be identified. To date, ADCC by NK cells has rarely been visualized in mouse models of tumortargeting $\mathrm{mAb}$ treatments despite being largely reported in in vitro studies. In one study using subcutaneously injected EL4 thymoma cells, Fc-mediated recognition of tumor-bound mAb by NK cells was not sufficient to induce target killing, but was required to stabilize interactions with target cells thus facilitating cell killing through the NKG2D activating receptor [51].

From another angle, bispecific antibodies have been engineered to promote contacts between tumors and effector cells. A recent study using a bispecific Ab targeting CD3 and the carcinoembryonic (CEA) tumor antigen in a colon carcinoma xenograft, confirmed that 
treatment with the bispecific $\mathrm{Ab}$ promoted $\mathrm{T}$ cell interactions with tumors, thus resulting in a greater cytotoxic activity at the tumor site [12].

\section{Immune checkpoint blockade.}

Immune checkpoint blockers (ICB), including mAb that target CTLA-4, PD-1 or PD-L1 (programmed death ligand 1), have shown great promise for the treatment of some cancers [52], yet a complete understanding of their in vivo modes of action is lacking.

In vivo imaging has revealed that ICB can have a strong impact on intratumoral $\mathrm{T}$ cell dynamics. Specifically, treatment with anti-CTLA-4 mAb appeared to increase overall melanoma-specific $\mathrm{CD}^{+} \mathrm{T}$ cell motility in the tumor microenvironment, although the underlying mechanism is unclear $[53,54]$. Another study reported that PD-1 blockade could restore CTL conjugation to target cells and their subsequent killing activity in lymph nodes in the context of graft versus leukemia effects [55]. Anti-PD-1 activity may also benefit from releasing $\mathrm{CD}^{+} \mathrm{T}$ cells engaged in unproductive interactions with macrophages as observed in a spontaneous murine model of breast cancer [31].

Imaging studies have also been key in uncovering unexpected aspects of anti-PD-1 therapy. For example, visualizing the fate of labeled anti-PD-1 mAb in mice bearing MC38 colon carcinomas revealed that after binding to $\mathrm{T}$ cells, these reagents are rapidly captured by neighboring macrophages in a Fc receptor-dependent manner [56]. Blocking this capture process increased anti-PD-1 activity by prolonging mAb binding time on $\mathrm{T}$ cells. Intratumoral imaging using reporters for IFN- $\gamma$ and IL-12 cytokines contributed to identify a key cellular crosstalk mediating anti-PD-1 therapeutic effects. In this model, IFN- $\gamma$ released by anti-PD-1sensitized $\mathrm{T}$ cells was essential to induce IL-12-production by DCs thereby increasing antitumor activity [11]. Notably, anti-PD-1 treatment might not only act to reduce tumor burden 
but might also finely tune its subclonal heterogeneity [20], a phenomenon that should be considered when designing combination therapies.

\section{Targeting macrophages.}

Targeting of tumor-associated macrophages (TAMs) may offer ways to release $\mathrm{T}$ cells and promote their dissemination in the tumor as mentioned previously. In addition, targeting macrophages using anti-CSF1 (colony stimulating factor 1) mAb was shown to restore tumor blood vessel patterning and function as visualized by longitudinal multiphoton imaging in a mouse glioma model [15]. Additionally, intravital confocal imaging was used to better characterize the impact of using depleting anti-CFS1R mAb on the myeloid cell compartment, revealing depletion of cells harboring markers of TAMs and DCs in a mouse model of breast cancer [57]. Finally, targeting the protease-dependent mesenchymal mode of migration of TAMs in a subcutaneous fibrosarcoma mouse model can limit their recruitment and subsequently reduce tumor growth [10].

\section{Cell therapy.}

Infusing expanded tumor-infiltrating lymphocytes (TILs) or genetically engineered $\mathrm{T}$ cells that express a tumor-specific TCR or a chimeric antigen receptor (CAR) can represent a very effective strategy to control tumor burden [58]. Among the important outstanding questions is how efficiently transferred effectors infiltrate the tumor and at which rate they kill tumor targets. Strategies to visualize tumor killing in real-time can provide insights into these important parameters. In a model of solid tumor using EL4 thymoma, adoptively transferred CTLs were found to kill tumor cells at a relatively low rate (1 killing event per CTL every 6hrs on average) and their accumulation at high density was essential to induce tumor regression [16]. Combining therapies might boost killing activity as illustrated by the use of an agonistic 
anti-CD137 (a T cell co-receptor) mAb which promoted the cytotoxic activity of transferred CTLs [59]. Notably, CTL activity can vary extensively depending on the anatomical site. In a model of graft versus leukemia effect, CTL recognition was specifically silenced in lymph nodes due to widespread PD-L1 upregulation as visualized by two-photon imaging [55]. CTL activity could be modulated over time as illustrated by the observation that their ability to stably engage targets decreased with their residence time in the tumor microenvironment [60].

Real-time imaging of CAR $\mathrm{T}$ cell killing and signaling using Förster resonance energy transfer (FRET)-based probes for apoptosis and calcium signals in a model of B cell lymphoma revealed extensive heterogeneity in CAR T cell activity [17]. Nevertheless, a subset of CAR T cells could engage, kill and detach from tumor targets in a short period of time (25 $\mathrm{min}$ ) and these events were sufficient to eliminate the bulk of tumor cells [17]. Finally, imaging may help validate strategies to overcome tumor escape from CAR T cell therapy such as the use of T cells expressing two distinct CARs directed against the CD19 and CD123 molecules that retain the ability to interact with CD19-negative leukemia cells [61].

Overall, intravital imaging not only improves our mechanistic understanding of these treatments but also provides clues about how to improve their activity in vivo.

\section{Concluding Remarks}

$\underline{\text { In sum, }}$ by providing dynamic information at the single-cell level in the tumor microenvironment, intravital imaging represents a powerful approach to identify fundamental mechanisms of tumor immunosurveillance and immunosuppression. In addition, this approach is refining, when not redefining, our understanding of how immunotherapies might actually be acting in vivo (Figure 3). This knowledge is essential to identify ways to improve or combine immunotherapies effectively. For this reason, one should consider including intravital imaging experiments as an essential step in preclinical development of new immunotherapies. 
Moreover, intravital imaging can be combined with other techniques. For instance, dynamic in situ cytometry (DISC) relies on cell surface labeling to link phenotypic markers and motility parameters [62]. Photoactivation of fluorescent proteins can be used to pinpoint some of the cells imaged and subsequently analyze them by flow cytometry [63], or single-cell RNA sequencing (Niche-Seq) [64]. Imaging can also be coupled to techniques that mark cell-cell interactions such as the LIPSTIC (Labeling Immune Partnerships by SorTagging Intercellular Contacts) approach [65]. Many new questions and challenges lie ahead (OUTSTANDING QUESTIONS). New mouse models of cancer relevant for human diseases, emerging immunotherapies as well as novel functional reporters and optogenetic strategies can be used to manipulate immune cells during imaging (Box 2); these approaches can further establish intravital imaging as a unique and essential tool enabling a deeper understanding of immune cell activities in the tumor microenvironment, and informing putative immunotherapeutic approaches to treating malignancies. 


\section{Box 1. Technical workflow for intravital imaging}

A typical intravital imaging experiment proceeds through the following steps:

\section{Choosing the relevant model to image}

- Select a genetically-engineered spontaneous or transplantable fluorescent mouse model of cancer

- Use fluorescent reporter recipients to visualize immune cells of interest and/or adoptively transfer fluorescently labeled immune cells

\section{Reaching the site of imaging}

- Insert window chamber for longitudinal imaging

- Surgically expose the site of interest for immediate imaging

\section{Image acquisition and data collection}

- Maintain optimal and physiological conditions for animal welfare and imaging quality: anesthesia, analgesia, temperature, oxygen...

- Choose the correct mirrors and filters to visualize fluorescent cells

- Choose the appropriate laser wavelength for the excitation of the distinct fluorescent proteins and dyes

- Determine acquisition parameters (laser power, 3D volume, scanning rate and time intervals)

- Define length of the recording

\section{Data extraction and analysis}

- Choose a relevant software for time-lapse movie analysis

- Determine the parameters to be quantified in the regions of interest (speed, confinement, shape, signal intensity, contacts...)

- Proceed to automated (eg. cell tracking) or manual parameter quantification

- Graph the extracted data

- Assemble the time-lapse movie as 2D projection of the 3D volume (legends, time display, scale bar) 


\section{Box 2. Combining intravital imaging and optogenetics to manipulate individual immune}

cells

The use of light sensitive-proteins has allowed the engineering of multiple genetically-encoded systems to manipulate cellular function with exquisite spatiotemporal control (referred to as actuators) [66,67]. The technologies, termed optogenetics have been used extensively in neuroscience but have only emerged recently as a new tool for immunologists. For example, optogenetic tools have already been used to manipulate trafficking of immune cells in vivo, by using a system in which CXCR4 signal is triggered by light exposure [68]. Calcium actuators that trigger aggregation of stromal interaction molecule 1 (STIM1) can provoke DC activation or augment $\mathrm{CD}^{+} \mathrm{T}$ cell cytotoxicity $[69,70]$. Chemokine secretion [71] or TCR signaling [72,73] can also be manipulated with light. To date, most optogenetic approaches with immune cells have been conducted in vitro or in vivo by bulk photoactivation of the whole tumor, but combined with intravital imaging, optogenetic actuators might also potentially allow the manipulation of single immune cells as we observe them. This may offer a unique ability to understand the functional contribution of specific cells and/or the consequence of a given cellular function in the tumor microenvironment. Among the challenges facing the use of single-cell optogenetics in tumors are the ability to design actuators with low background activity and high response rates, to introduce actuators in primary immune cells and to photoactivate cells located deeply inside tumors. Yet, controlling single immune cells in tumors and assessing the functional consequences of such manipulations may represent an important milestone in the field of in intravital imaging. 


\section{Glossary}

Actuators: Genetically-encoded tools for light-activated control of proteins regulating cellular functions.

ADCC: A cytotoxic immune response often elicited by NK cells following the recognition through Fc receptors of antibody-bound tumor cells. This results in the delivery of their cytotoxic content toward the target cell and its subsequent death.

Adoptive cell transfer: A therapy based on the isolation and the infusion of immune cells into the same or another individual.

ADP: The mechanism by which macrophages can recognize, through their Fc receptors, antibody bound to tumor cells, resulting in cell engulfment and destruction.

B cell lymphoma: B cell-derived tumors developing in lymphoid organs.

Bispecific antibodies: Synthetic antibodies that can simultaneously bind two distinct antigens.

CAR T: T cells genetically engineered to express synthetic receptors composed of intracellular signaling domains fused to an antibody-derived recognition domain specific for surface target antigens.

CDC: A lysis mechanism triggered by the recruitment of molecules of the complement pathway upon antibody binding to its target cells.

DISC: A technique combining advantages of intravital imaging and flow cytometry analysis to link cell behavior and phenotype in vivo.

Ectopic transplantation: Transplantation in a different anatomical site than the original location in the donor.

E $\boldsymbol{\mu}$-myc mice: Genetically modified mice that express the oncogene c-myc driven by the immunoglobulin heavy chain enhancer and that develop spontaneous aggressive B-cell lymphoma. 
FRET: An energy transfer between a pair of adjacent fluorophores. FRET is used to monitor proximity between protein domains, conformational changes or protein cleavage.

Graft versus leukemia effect: An immune response against host leukemic cells mediated by transplanted donor immune cells.

ICB: A strategy that aims at increasing immune responses through the blockade of immunoreceptors that inhibit the activity of immune effectors.

Kupffer cells: Resident macrophages found in the liver.

LIPSTIC: A technique that enables the identification of receptor-ligand interactions between cells by using an intercellular enzymatic labeling. When a ligand and its receptor interact, the enzyme mediates the transfer of a substrate onto the tagged receptor.

MMTV-PyMT model: A transgenic mouse model of breast cancer. The mammary tumor virus long terminal repeat (MMTV-LTR) drives the expression of mammary gland specific polyomavirus middle $\mathrm{T}$ antigen (PyMT), resulting in a rapid development of highly metastatic tumors.

Monoclonal antibody: A specific antibody that recognizes a unique epitope.

Multicolor barcoding: A series of approach to randomly label individual tumor cells with one of multiple fluorescent proteins to follow subclonal progenies. Barcoding can be performed ex vivo by transducing tumor cells prior to transfer or directly in vivo using Cre-based random genetic recombination in transgenic mouse models.

NETs: DNA meshes associated to cytotoxic enzymes that are released in the extracellular space by neutrophils and that can trap, neutralize and kill pathogens.

Niche-Seq: A technique that uses photoactivatable fluorescent proteins to sort and analyze gene expression of cells located in selected areas of specific tissue.

NKG2D: An activating receptor expressed by NK and T cells. 
Orthotopic transplantation: Transplantation in the same anatomical site where cells or tissue were originally extracted from the donor.

STIM1: A transmembrane protein, mainly localized in the endoplasmic reticulum, involved in the control of calcium entry within the intracellular space.

Syngeneic: Sharing the same genetic background.

TILs: Lymphocytes localized in the tumor microenvironment.

Transmigration: Migration of cells across the endothelium vessel wall.

Tregs: A subset of $\mathrm{T}$ cells specialized in the suppression of $\mathrm{T}$ cell responses.

Tumor microenvironment: The environment that surrounds and feeds tumor cells. This includes blood vessels, immune cells, fibroblasts, extracellular matrix and signaling molecules. Tumor microparticles: Microscale blebs that shed from a tumor cell.

Two-photon microscopy: A fluorescence imaging technique that uses two photons of lowenergy infrared light to excite fluorophores. This greatly improves imaging depth compared to confocal microscopy and reduces phototoxic effects associated with visible light.

Window chamber: Glass-covered structure surgically implanted into the recipient to enable repeated imaging of a tissue by intravital microscopy.

Xenograft: A tissue graft originating from a different species than the recipient. 


\section{Acknowledgments}

We thank Mikael Pittet, Thorsten Mempel, Thomas Gebhardt for kindly providing images. The work was supported by Institut Pasteur, Inserm and an Advanced grant (ENLIGHTEN) from the European Research Council (P.B). 


\section{References}

1 Wei, S.C. et al. (2017) Distinct Cellular Mechanisms Underlie Anti-CTLA-4 and AntiPD-1 Checkpoint Blockade. Cell 170, 1120-1133.e17

2 Gordon, S. (2008) Elie Metchnikoff: Father of natural immunity. European Journal of Immunology 38, 3257-3264

3 Ritsma, L. et al. (2012) Intravital imaging of cell signaling in mice. IntraVital 1, 2-10

4 Secklehner, J. et al. (2017) Intravital microscopy in historic and contemporary immunology. Immunol Cell Biol 95, 506-513

5 Ellenbroek, S.I.J. and Rheenen, J. van (2014) Imaging hallmarks of cancer in living mice. Nature Reviews Cancer 14, 406-418

6 Fukumura, D. et al. (2010) Tumor Microvasculature and Microenvironment: Novel Insights Through Intravital Imaging in Pre-Clinical Models. Microcirculation 17, 206-225

7 Guy, C.T. et al. (1992) Induction of mammary tumors by expression of polyomavirus middle T oncogene: a transgenic mouse model for metastatic disease. Molecular and Cellular Biology 12, 954-961

8 Adams, J.M. et al. (1985) The c- myc oncogene driven by immunoglobulin enhancers induces lymphoid malignancy in transgenic mice. Nature 318, 533

9 Harris, A.W. et al. (1988) The E mu-myc transgenic mouse. A model for high-incidence spontaneous lymphoma and leukemia of early B cells. Journal of Experimental Medicine 167, $353-371$

10 Gui, P. et al. (2018) The Protease-Dependent Mesenchymal Migration of TumorAssociated Macrophages as a Target in Cancer Immunotherapy. Cancer Immunol Res 6, 13371351

11 Garris, C.S. et al. (2018) Successful Anti-PD-1 Cancer Immunotherapy Requires T 
Cell-Dendritic Cell Crosstalk Involving the Cytokines IFN- $\gamma$ and IL-12. Immunity 49, 11481161.e7

12 Lehmann, S. et al. (2016) In Vivo Fluorescence Imaging of the Activity of CEA TCB, a Novel T-Cell Bispecific Antibody, Reveals Highly Specific Tumor Targeting and Fast Induction of T-Cell-Mediated Tumor Killing. Clin Cancer Res 22, 4417-4427

13 Headley, M.B. et al. (2016) Visualization of immediate immune responses to pioneer metastatic cells in the lung. Nature 531, 513-517

14 Jung, K. et al. (2017) Ly6C $\mathrm{C}^{\text {lo }}$ monocytes drive immunosuppression and confer resistance to anti-VEGFR2 cancer therapy. J Clin Invest 127, 3039-3051

15 Mathivet, T. et al. (2017) Dynamic stroma reorganization drives blood vessel dysmorphia during glioma growth. EMBO Molecular Medicine 9, 1629-1645

16 Breart, B. et al. (2008) Two-photon imaging of intratumoral $\mathrm{CD}^{+} \mathrm{T}$ cell cytotoxic activity during adoptive T cell therapy in mice. J Clin Invest 118, 1390-1397

17 Cazaux, M. et al. (2019) Single-cell imaging of CAR T cell activity in vivo reveals extensive functional and anatomical heterogeneity. Journal of Experimental Medicine DOI: 10.1084/jem.20182375

18 Yano, S. and Hoffman, R.M. (2018) Real-Time Determination of the Cell-Cycle Position of Individual Cells within Live Tumors Using FUCCI Cell-Cycle Imaging. Cells 7, 168

19 Zomer, A. et al. (2013) Brief Report: Intravital Imaging of Cancer Stem Cell Plasticity in Mammary Tumors. Stem Cells 31, 602-606

20 Milo, I. et al. (2018) The immune system profoundly restricts intratumor genetic heterogeneity. Science Immunology 3, eaat1435

21 Marangoni, F. et al. (2013) The Transcription Factor NFAT Exhibits Signal Memory during Serial T Cell Interactions with Antigen-Presenting Cells. Immunity 38, 237-249 
22 Conway, J.R.W. et al. (2014) Developments in preclinical cancer imaging: innovating the discovery of therapeutics. Nature Reviews Cancer 14, 314-328

23 Boissonnas, A. et al. (2007) In vivo imaging of cytotoxic T cell infiltration and elimination of a solid tumor. Journal of Experimental Medicine 204, 345-356

24 Mrass, P. et al. (2006) Random migration precedes stable target cell interactions of tumor-infiltrating T cells. Journal of Experimental Medicine 203, 2749-2761

25 Mrass, P. et al. (2008) CD44 Mediates Successful Interstitial Navigation by Killer T Cells and Enables Efficient Antitumor Immunity. Immunity 29, 971-985

26 Park, S.L. et al. (2019) Tissue-resident memory CD8 + T cells promote melanomaimmune equilibrium in skin. Nature 565, 366-371

27 Deguine, J. et al. (2010) Intravital Imaging Reveals Distinct Dynamics for Natural Killer and CD8+ T Cells during Tumor Regression. Immunity 33, 632-644

28 Salmon, H. et al. (2012) Matrix architecture defines the preferential localization and migration of T cells into the stroma of human lung tumors. J Clin Invest 122, 899-910

29 Peranzoni, E. et al. (2017) Ex Vivo Imaging of Resident CD8 T Lymphocytes in Human Lung Tumor Slices Using Confocal Microscopy. JoVE (Journal of Visualized Experiments) DOI: $10.3791 / 55709$

30 Bougherara, H. et al. (2015) Real-Time Imaging of Resident T Cells in Human Lung and Ovarian Carcinomas Reveals How Different Tumor Microenvironments Control T Lymphocyte Migration. Front. Immunol. 6,

31 Peranzoni, E. et al. (2018) Macrophages impede CD8 T cells from reaching tumor cells and limit the efficacy of anti-PD-1 treatment. PNAS 115, E4041-E4050

32 Boissonnas, A. et al. (2013) CD8+ Tumor-Infiltrating T Cells Are Trapped in the Tumor-Dendritic Cell Network. Neoplasia 15, 85-94

33 Moalli, F. et al. (2015) Intravital and Whole-Organ Imaging Reveals Capture of 
Melanoma-Derived Antigen by Lymph Node Subcapsular Macrophages Leading to Widespread Deposition on Follicular Dendritic Cells. Front. Immunol. 6,

34 Engelhardt, J.J. et al. (2012) Marginating Dendritic Cells of the Tumor Microenvironment Cross-Present Tumor Antigens and Stably Engage Tumor-Specific T Cells. Cancer Cell 21, 402-417

35 Broz, M.L. et al. (2014) Dissecting the Tumor Myeloid Compartment Reveals Rare Activating Antigen-Presenting Cells Critical for T Cell Immunity. Cancer Cell 26, 638-652 36 Barry, K.C. et al. (2018) A natural killer-dendritic cell axis defines checkpoint therapyresponsive tumor microenvironments. Nature Medicine 24, 1178

37 Mempel, T.R. et al. (2006) Regulatory T Cells Reversibly Suppress Cytotoxic T Cell Function Independent of Effector Differentiation. Immunity 25, 129-141

38 Boissonnas, A. et al. (2010) Foxp3+ T Cells Induce Perforin-Dependent Dendritic Cell Death in Tumor-Draining Lymph Nodes. Immunity 32, 266-278

39 Bauer, C.A. et al. (2014) Dynamic Treg interactions with intratumoral APCs promote local CTL dysfunction. J Clin Invest 124, 2425-2440

40 Jang, J.-E. et al. (2017) Crosstalk between Regulatory T Cells and Tumor-Associated Dendritic Cells Negates Anti-tumor Immunity in Pancreatic Cancer. Cell Reports 20, 558-571 41 Qi, S. et al. (2016) Long-term intravital imaging of the multicolor-coded tumor microenvironment during combination immunotherapy. eLife 5, e14756

42 Rytelewski, M. et al. (2019) Merger of dynamic two-photon and phosphorescence lifetime microscopy reveals dependence of lymphocyte motility on oxygen in solid and hematological tumors. Journal for ImmunoTherapy of Cancer 7, 78

43 Arlauckas, S.P. et al. (2018) Arg1 expression defines immunosuppressive subsets of tumor-associated macrophages. Theranostics 8, 5842-5854

44 Harney, A.S. et al. (2015) Real-Time Imaging Reveals Local, Transient Vascular 
Permeability, and Tumor Cell Intravasation Stimulated by TIE2hi Macrophage-Derived VEGFA. Cancer Discov 5, 932-943

45 Park, J. et al. (2016) Cancer cells induce metastasis-supporting neutrophil extracellular DNA traps. Science Translational Medicine 8, 361ra138-361ra138

46 Reichert, J.M. and Dhimolea, E. (2012) The future of antibodies as cancer drugs. Drug Discovery Today 17, 954-963

47 Weiner, L.M. et al. (2010) Monoclonal antibodies: versatile platforms for cancer immunotherapy. Nature Reviews Immunology 10, 317-327

48 Montalvao, F. et al. (2013) The mechanism of anti-CD20-mediated B cell depletion revealed by intravital imaging. $J$ Clin Invest $123,5098-5103$

49 Gül, N. et al. (2014) Macrophages eliminate circulating tumor cells after monoclonal antibody therapy. $J$ Clin Invest $124,812-823$

50 Grandjean, C.L. et al. (2016) Intravital imaging reveals improved Kupffer cell-mediated phagocytosis as a mode of action of glycoengineered anti-CD20 antibodies. Scientific Reports 6,34382

51 Deguine, J. et al. (2012) Cutting Edge: Tumor-Targeting Antibodies Enhance NKG2DMediated NK Cell Cytotoxicity by Stabilizing NK Cell-Tumor Cell Interactions. The Journal of Immunology 189, 5493-5497

52 Seidel, J.A. et al. (2018) Anti-PD-1 and Anti-CTLA-4 Therapies in Cancer: Mechanisms of Action, Efficacy, and Limitations. Front. Oncol. 8,

53 Pentcheva-Hoang, T. et al. (2014) Cytotoxic T Lymphocyte Antigen-4 Blockade Enhances Antitumor Immunity by Stimulating Melanoma-Specific T-cell Motility. Cancer Immunol Res 2, 970-980

54 Ruocco, M.G. et al. (2012) Suppressing T cell motility induced by anti-CTLA-4 monotherapy improves antitumor effects. J Clin Invest 122, 3718-3730 
55 Michonneau, D. et al. (2016) The PD-1 Axis Enforces an Anatomical Segregation of CTL Activity that Creates Tumor Niches after Allogeneic Hematopoietic Stem Cell Transplantation. Immunity 44, 143-154

56 Arlauckas, S.P. et al. (2017) In vivo imaging reveals a tumor-associated macrophage mediated resistance pathway in anti-PD-1 therapy. Sci Transl Med 9,

57 Lohela, M. et al. (2014) Intravital imaging reveals distinct responses of depleting dynamic tumor-associated macrophage and dendritic cell subpopulations. PNAS 111, E5086E5095

58 June, C.H. et al. (2014) Engineered T cells for cancer therapy. Cancer Immunol Immunother 63, 969-975

59 Weigelin, B. et al. (2015) Focusing and sustaining the antitumor CTL effector killer response by agonist anti-CD137 mAb. PNAS 112, 7551-7556

60 Boldajipour, B. et al. (2016) Tumor-infiltrating lymphocytes are dynamically desensitized to antigen but are maintained by homeostatic cytokine. JCI Insight 1 ,

61 Ruella, M. et al. (2016) Dual CD19 and CD123 targeting prevents antigen-loss relapses after CD19-directed immunotherapies. J Clin Invest 126, 3814-3826

62 Moreau, H.D. et al. (2012) Dynamic In Situ Cytometry Uncovers T Cell Receptor Signaling during Immunological Synapses and Kinapses In Vivo. Immunity 37, 351-363

63 Victora, G.D. et al. (2010) Germinal Center Dynamics Revealed by Multiphoton Microscopy with a Photoactivatable Fluorescent Reporter. Cell 143, 592-605

64 Medaglia, C. et al. (2017) Spatial reconstruction of immune niches by combining photoactivatable reporters and scRNA-seq. Science 358, 1622-1626

65 Pasqual, G. et al. (2018) Monitoring T cell-dendritic cell interactions in vivo by intercellular enzymatic labelling. Nature 553, 496-500

66 Gautier, A. et al. (2014) How to control proteins with light in living systems. Nature 
Chemical Biology 10, 533-541

67 Tischer, D. and Weiner, O.D. (2014) Illuminating cell signalling with optogenetic tools. Nature Reviews Molecular Cell Biology 15, 551-558

$68 \mathrm{Xu}, \mathrm{Y}$. et al. (2014) Optogenetic control of chemokine receptor signal and T-cell migration. PNAS 111, 6371-6376

69 He, L. et al. (2015) Near-infrared photoactivatable control of Ca2+ signaling and optogenetic immunomodulation. eLife 4, e10024

70 Kim, K.-D. et al. (2017) Targeted calcium influx boosts cytotoxic T lymphocyte function in the tumour microenvironment. Nature Communications 8, 15365

71 Sarris, M. et al. (2016) Manipulating leukocyte interactions in vivo through optogenetic chemokine release. Blood 127, e35-e41

72 Yousefi, O.S. et al. (2019) Optogenetic control shows that kinetic proofreading regulates the activity of the $\mathrm{T}$ cell receptor. eLife 8 , e 42475

73 Tischer, D.K. and Weiner, O.D. (2019) Light-based tuning of ligand half-life supports kinetic proofreading model of T cell signaling. eLife 8, e42498

74 Thestrup, T. et al. (2014) Optimized ratiometric calcium sensors for functional in vivo imaging of neurons and T lymphocytes. Nature Methods 11, 175-182

75 Snippert, H.J. et al. (2010) Intestinal Crypt Homeostasis Results from Neutral Competition between Symmetrically Dividing Lgr5 Stem Cells. Cell 143, 134-144 
Table 1. Tumor models, anatomical sites and immune cells used in intravital imaging studies.

\begin{tabular}{|c|c|c|c|c|}
\hline $\begin{array}{c}\text { Site of } \\
\text { imaging }\end{array}$ & Tumor model & Immune cells & Window chamber & Refs. \\
\hline \multirow{15}{*}{ Skin } & \multirow{3}{*}{ EL4 or EG7 $\left(\mathrm{OVA}^{+}\right)$murine thymoma s.c. } & $\mathrm{T}$ & & {$[16,23-25]$} \\
\hline & & NK and $\mathrm{T}$ & & [27] \\
\hline & & NK & & [51] \\
\hline & B16 murine melanoma s.c or epicutaneous & $\mathrm{T}_{\mathrm{RM}}$ & & [26] \\
\hline & \multirow{2}{*}{ B16 murine melanoma s.c. } & Tregs, CTL, DC, N & & {$[41]$} \\
\hline & & CTL & & {$[53,59]$} \\
\hline & B78 murine melanoma i.d. & $\mathrm{NK}, \mathrm{DC}$ & & [36] \\
\hline & 4T1 murine breast cancer s.c. & CTL & & {$[54]$} \\
\hline & MCA-OVA murine fibroscarcoma s.c. & CTL, DC & & {$[32]$} \\
\hline & LBP murine fibrosarcoma s.c. & TAM & Dorsal window & {$[10]$} \\
\hline & \multirow{2}{*}{ CT26 murine colon carcinoma s.c. } & CTL & & [21] \\
\hline & & CTL, Tregs, APC & & [39] \\
\hline & \multirow[t]{2}{*}{ MC38 murine colon cancer s.c. } & $\mathrm{T}, \mathrm{TAM}$ & & {$[43,56]$} \\
\hline & & $\mathrm{T}, \mathrm{DC}$ & Dorsal skinfold & [11] \\
\hline & Human colorectal cancer CEA ${ }^{+}$s.c. & $\mathrm{T}$ & Dorsal skinfold & {$[12]$} \\
\hline \multirow{3}{*}{ Lung } & 4T1 murine breast cancer orthotopically & $\mathrm{N}$ & & {$[45]$} \\
\hline & MCA-205 murine fibrosarcoma cells i.v. & $\mathrm{T}$ & & [42] \\
\hline & B16.F10 murine melanoma i.v. & $\mathrm{N}, \mathrm{Mo}, \mathrm{DC}$ & Intercostal window & [13] \\
\hline \multirow{3}{*}{$\begin{array}{c}\text { Mammary } \\
\text { fatpads }\end{array}$} & \multirow{3}{*}{ Spontaneous breast tumors in MMTV-PyMT mice } & $\mathrm{T}, \mathrm{DC}$ & & [35] \\
\hline & & TAM & & {$[44]$} \\
\hline & & TAM, DC & & [57] \\
\hline Pancreas & $\mathrm{Kras}^{\mathrm{G} 12 \mathrm{D}}$-PDEC orthotopically & CTL, Tregs, DC & & {$[40]$} \\
\hline \multirow{4}{*}{ Lymph nodes } & B16.F0 murine melanoma s.c. & TAM, FDC, B & & [33] \\
\hline & CT44 murine colon carcinoma f.p & CTL, Tregs, B & & [37] \\
\hline & MCA-OVA murine fibroscarcoma s.c. & CTL, Tregs, DC & & [38] \\
\hline & E $\mu$-myc murine B-cell lymphoma i.v. & CTL & & {$[55]$} \\
\hline \multirow{4}{*}{ Bone marrow } & \multirow{2}{*}{ E $\mu$-myc murine B-cell lymphoma i.v. } & CTL & & {$[20]$} \\
\hline & & CAR T & & {$[17]$} \\
\hline & Human B-cell acute leukemia xenograft i.v. & CAR T & & {$[61]$} \\
\hline & Spontaneous B-cell acute leukemia BCR-ABL ${ }^{+}$ & $\mathrm{T}$ & & {$[42]$} \\
\hline Liver & E $\mu$-myc murine B-cell lymphoma i.v. & $\mathrm{KC}$ & & {$[48,50]$} \\
\hline Kidney & EL4 murine thymoma i.v. & CTL, DC & & [32] \\
\hline Cecum & SL4 or CT26 murine colorectal cancer orthotopically & Mo & Cecum window & [14] \\
\hline Cranial & CT2A and GL261 murine glioma intracranially & TAM & Cranial window & [15] \\
\hline \multirow{3}{*}{$\begin{array}{c}\text { Human } \\
\text { cancer slices }\end{array}$} & Breast cancer & TAM & & {$[10]$} \\
\hline & Lung cancer & TAM, CTL & & {$[29,31]$} \\
\hline & Ovarian and lung cancer & CTL & & {$[30]$} \\
\hline
\end{tabular}


s.c.: subcutaneous; i.d.: intradermal; i.v.: intravenous; f.p.: footpad; T: T cells; B: B cells; CTL: cytotoxic T lymphocytes; $\mathrm{T}_{\mathrm{RM}}$ : tissue-resident memory $\mathrm{T}$ cells; Tregs: regulatory $\mathrm{T}$ lymphocytes; $\mathrm{CAR} \mathrm{T}$ : chimeric antigen receptor T cells; NK: natural killer cell; APC: antigen-presenting cell; DC: dendritic cell; FDC: follicular dendritic cells; TAM: tumor-associated macrophages; Mo: monocytes; N: neutrophils; KC: Kupffer cells. 


\section{Figure legends}

Figure 1, Key Figure. Intravital imaging provides access to dynamic parameters within the tumor microenvironment.

This figure illustrates the diversity of parameters that can be collected using two-photon microscopy. Cell migration, cellular interactions, cell division or phagocytosis are readily visualized using fluorescently-labeled cells but the introduction of functional reporters has extended the list of measurable parameters including cell signaling, cell death or gene expression. For instance, nuclear translocation of nuclear factor of activated T cells (NFAT) [21] and calcium $\left(\mathrm{Ca}^{2+}\right)$ probes $[17,74]$ have been used as readouts of TCR signaling and signal transduction respectively. Cytokine gene expression can be monitored using for example interferon gamma (IFN- $\gamma$ ) or interleukin 12 (IL-12) reporters [11]. A fluorescent probe reporting caspase-3 activity enables the detection of apoptosis in real time and in vivo [16,17]. Combining several of these readouts can provide unique spatiotemporal clues on immune and tumor cell activity within the tumor microenvironment.

Figure 2. Examples of genetically-encoded fluorescent reporters for the study of hosttumor interactions.

The left column depicts examples of reporters expressed by tumor cells. Tumor apoptosis has been visualized in real-time using a Förster resonance energy transfer (FRET)-based reporter for caspase-3 activity. Cyan fluorescent protein (CFP) and yellow fluorescent protein (YFP) are linked by the DEVD peptide, which is cleaved upon caspase-3 activation, resulting in loss of FRET in apoptotic cells [16,17]. Based on genetic recombination [19] or on multicolor ex vivo labeling of tumor cells [20], multicolor barcoding strategies have been employed to investigate intratumoral spatial architecture and clonal heterogeneity. As example for genetic 
recombination, a multicolor reporter is depicted [75]. Upon Cre recombinase activation, various fluorescent proteins (nuclear green fluorescent protein (GFP), YFP, red fluorescent protein (RFP) and membrane CFP) are randomly expressed. A proliferation sensor, called fluorescence ubiquitination cell cycle indicator (FUCCI) enabled visualization of cell-cycle dynamics in realtime in individual tumor cells [18].

The right column shows some reporters expressed by immune cells. Fluorescent sensors for calcium signaling can be used to detect productive T cell contacts. An example is the Twitch2B calcium indicator in which FRET occurs upon calcium-binding to troponin C (TnC) [17,74]. Reporter mice have been developed to monitor gene activation at the single-cell level. For example, IL-12 and IFN- $\gamma$ reporters use an internal ribosome entry site (IRES) to express enhanced YFP (eYFP) following cytokine gene activation [11]. Finally, nuclear translocation of NFAT-GFP has been used as a readout of TCR signaling [21].

Figure 3. Examples of the use of intravital imaging to assess mechanisms of immunosurveillance or the effects of immunotherapies in vivo in mice.

This figure compiles time-lapse images from different studies illustrating mechanisms of intratumoral immune activity identified by intravital two-photon imaging. Images are representative of A) Immunosuppression by regulatory $\mathrm{T}$ cells (Tregs) forming transient contacts with antigen-presenting cells (APCs) as visualized by two-photon imaging using a dorsal skin-fold chamber. Scale bar, $10 \mu \mathrm{m}$. Adapted from [39]. B) A crosstalk during antiPD-1 mAb therapy involves IL-12-producing dendritic cells and IFN- $\gamma$-producing T cells as visualized by two-photon microscopy using cytokine reporter mice. Arrows highlight IL12p40-eYFP-expressing cells. TAM, tumor-associated macrophages. Scale bar, $30 \mu \mathrm{m}$. Adapted from [11]. C) In vivo two-photon imaging has been used to track the fate of antiPD-1 mAb (aPD-1) revealing rapid capture by TAM. Yellow arrows indicate sites of anti- 
PD-1 mAb binding to $\mathrm{CD}^{+} \mathrm{T}$ cells at $15 \mathrm{~min}$. At $30 \mathrm{~min}$, anti-PD-1 $\mathrm{mAb}$ is internalized in

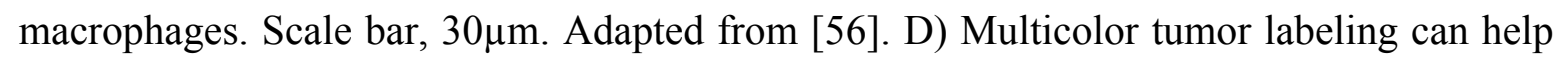
visualize the extent of clonal heterogeneity in a developing lymphoma in the bone marrow. Scale bar, $50 \mu \mathrm{m}$. Adapted from [20]. E) Visualizing tumor cell apoptosis can reveal rapid and direct killing activity of chimeric antigen receptor (CAR) T cells in the bone marrow. Red arrowheads show a CAR T cell coming into contact with a tumor cell (dotted circle) that

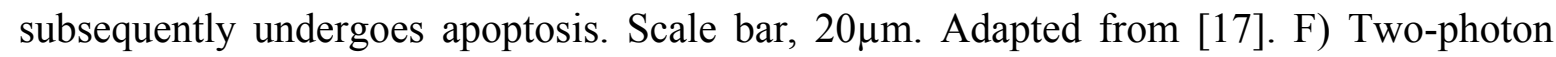
imaging after treatment with an anti-CD20 antibody highlights rapid and efficient B cell phagocytosis by the resident macrophages of the liver. Scale bar, $25 \mu \mathrm{m}$. Adapted from [50]. G) Time-lapse images reveal tissue-resident memory $T\left(T_{R M}\right)$ cells dynamically surveying B16 melanoma cells in the skin. Second harmonic generation (SHG) is a label-free signal that can help visualize collagen fibers. Scale bar, $25 \mu \mathrm{m}$. Adapted from [26]. H) Cytotoxic $\mathrm{CD}^{+} \mathrm{T}$ lymphocytes and NK cells exhibit distinct behaviors during target cell killing in lymph nodes. Individual micrographs (effectors in green, targets in red) are shown for cells establishing contacts, thus revealing that $\mathrm{CD}^{+} \mathrm{T}$ cells maintain contacts with moving targets (superimposed tracks) whereas NK cells transiently interact with their targets (intercrossed tracks). Scale bar, $40 \mu \mathrm{m}$. Adapted from [27]. 


\section{Tumor cells}

\section{Cell death} sensor

eg.reporter of caspase 3 activity
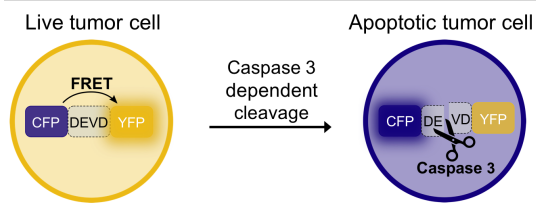

\section{Multicolor barcoding}

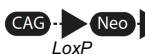

LoxP

\section{Genetic}

recombination

Multicolor ex

vivo labeling

\section{Proliferation sensor}

FUCCI

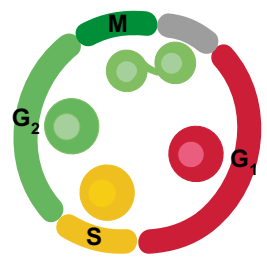

\section{Calcium} sensor

\section{Immune cells}

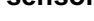

eg. Twitch

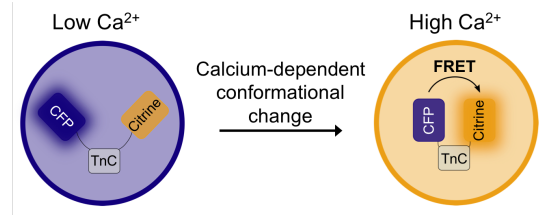

\section{Cytokine reporter}

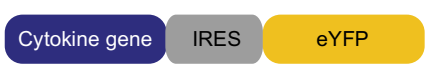

eg. IL-12-eYFP

IFN- $\gamma$-eYFP

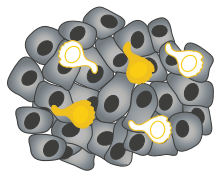

\section{Signaling} reporter

eg. NFAT-GFP

nuclear

translocation

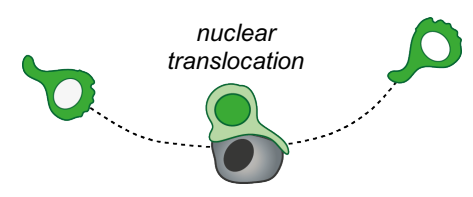


A. Defining mechanisms of immunosuppression

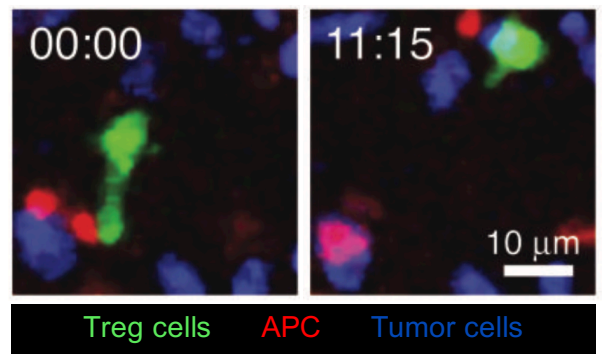

H. Unraveling specific behavior of tumor-infiltrating immune cells

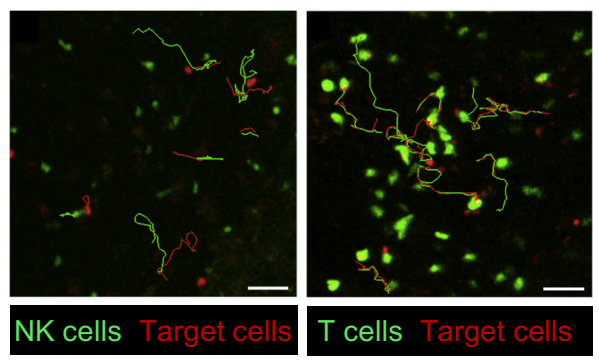

G. Characterizing actors of immunosurveillance

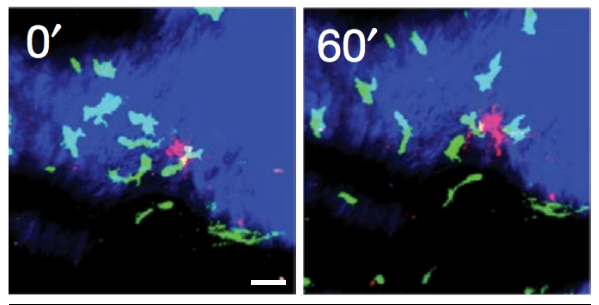

$\mathrm{T}_{\mathrm{RM}}$ cells
B. Uncovering cellular crosstalk
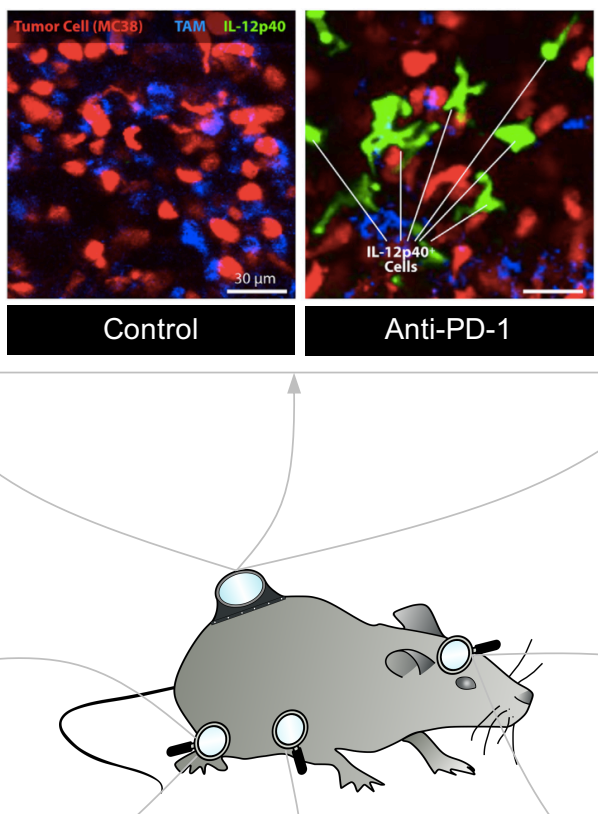

F. Identifying cellular effectors during immunotherapy

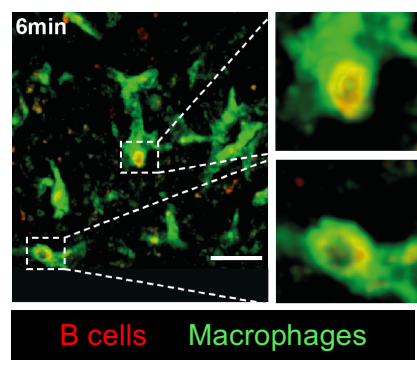

C. Tracking the fate of immunotherapeutic drug

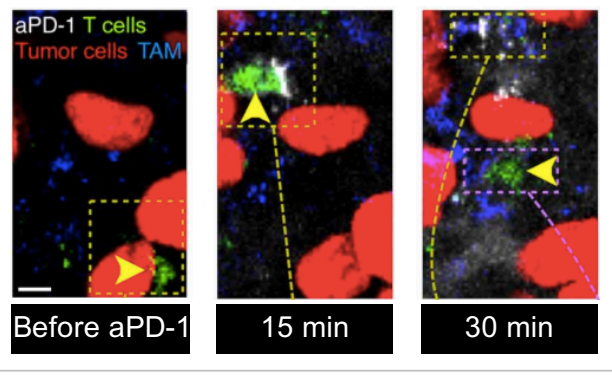

D. Revealing intratumoral clonal heterogeneity

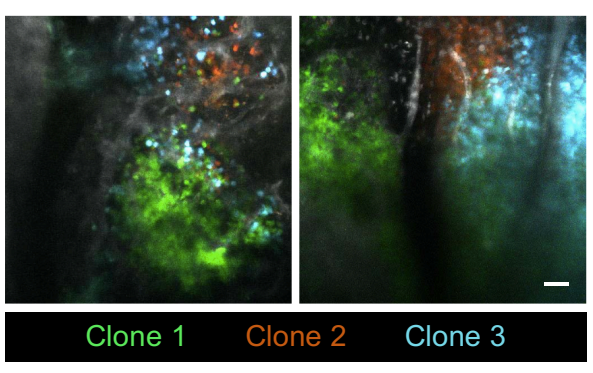

E. Determining immune antitumor activity

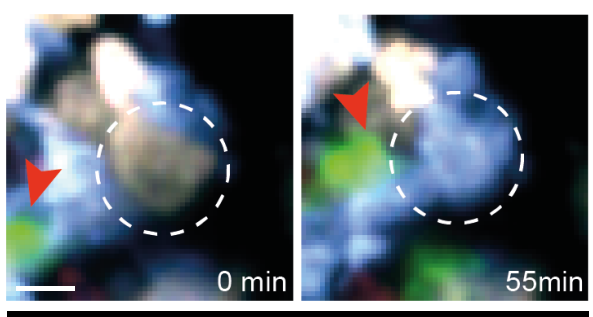

CAR T cells Live tumors 